\title{
Application of High Temperature Superconductor in National Institute for Fusion Science
}

T. Mito, H. Chikaraishi, S. Hamaguchi, R. Heller, S. Imagawa, K. Ishibashi, M. Iwakuma, A. Iwamoto, K. Maehata, R. Maekawa, J. Morikawa, O. Motojima, A. Nishimura, Y. Ogawa, T. Satow, T. Shintomi, K. Tachikawa, K. Takahata, H. Tamura, S. Yamada, Y. Yamada, and N. Yanagi

\begin{abstract}
In the National Institute for Fusion Science (NIFS), the following three research items are being advanced as application of high temperature superconductor (HTS) to an experimental fusion plasma system, through collaboration with universities and laboratories. The first is an application of HTS in the large current feedthrough to a superconducting coil cooled with superfluid helium in order to reconcile low heat load and stable large current transport capability. A $20 \mathrm{kA}$ current feedthrough from $4.4 \mathrm{~K}$ to $1.8 \mathrm{~K}$ has been being developed using YBCO bulk superconductors manufactured by the QMG process. The second is the development of a $30 \mathrm{kA}$ HTS current lead. The Bi2212 bulk material fabricated by the diffusion process is considered as a candidate material for HTS current leads. The third is the development of the superconducting magnetic-levitation coil for Mini-RT wound with Bi2223 tape conductor. A magnetic-levitation coil operated in persistent-current mode has to levitate for 8 hours without a feed of refrigerant. Present status of these HTS application researches in NIFS is reported.
\end{abstract}

Index Terms-Bi2212, Bi2223, current feedthrough, current lead, HTS, magnetic-levitation coil, mini-RT, YBCO.

\section{INTRODUCTION}

A FTER the discovery of high temperature superconductor (HTS), it was expected that various superconducting applications will expand rapidly using advantages of HTS due to the high operating temperature, such as simplification of cryogenic system, improvement in stability, etc. However, from the discovery of this new material, much time has been necessary until the utilization. Similarly the application of a low temperature superconductor (LTS) took a long time. When we try to apply a HTS to an experimental fusion plasma system, many subjects which should be overcome, such as mechanical weakness and a low critical current density in the magnetic field, are left behind. Also in it, the application of HTS as a large current

Manuscript received September 24, 2001. This work was supported in part by a Grant-in-Aid for Scientific Research 11210209.

T. Mito, H. Chikaraishi, S. Hamaguchi, S. Imagawa, A. Iwamoto, R. Maekawa, O. Motojima, A. Nishimura, T. Satow, K. Takahata, H. Tamura, S. Yamada, and N. Yanagi are with the NIFS National Institute for Fusion Science, 322-6 Oroshi-cho, Toki, Gifu 509-5292, Japan (e-mail: mito@nifs.ac.jp).

R. Heller is with FZK Forschungszentrum Karlsruhe, D-76021, Karlsruhe, Germany.

K. Ishibashi, M. Iwakuma, and K. Maehata are with Kyushu University, Fukuoka 812-8581, Japan.

J. Morikawa and Y. Ogawa are with The University of Tokyo, Bunkyo-ku, Tokyo 113-8656, Japan.

T. Shintomi is with the High Energy Accelerator Research Organization (KEK), Tsukuba, Ibaraki 305-0801, Japan.

K. Tachikawa and Y. Yamada are with Tokai University, Hiratsuka, Kanagawa 259-1292, Japan.

Publisher Item Identifier S 1051-8223(02)03615-1.

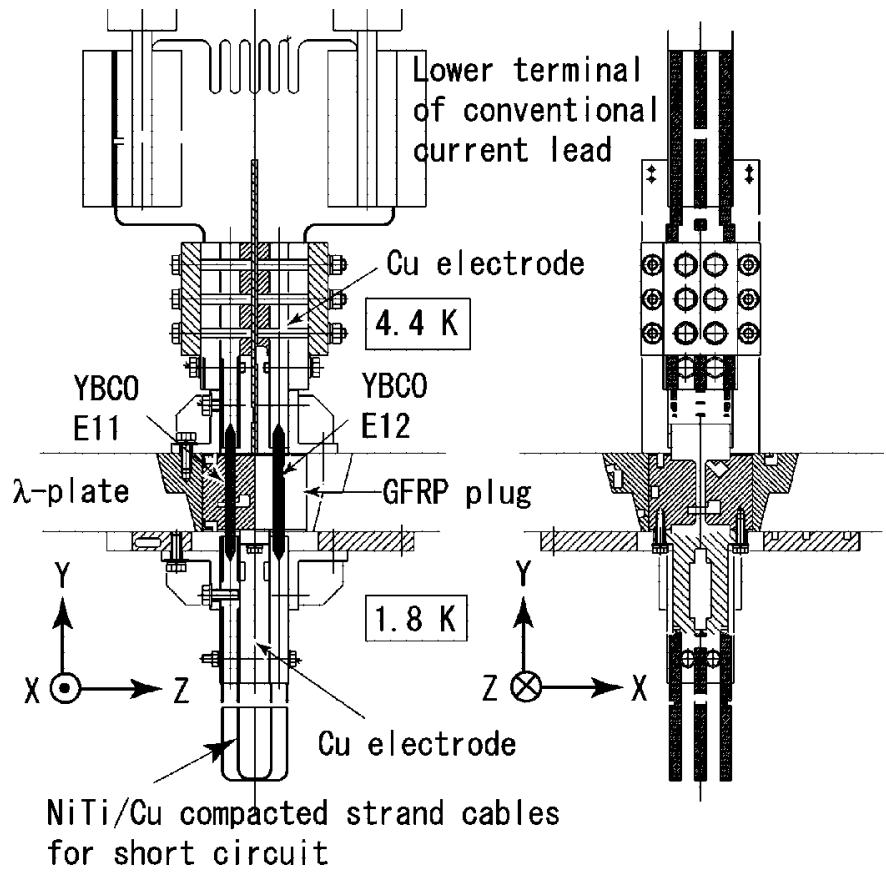

Fig. 1. Schematic drawing of the HTS current feedthrough: Prototype-I.

feeder to the LTS coil is considered to be an item most close to the practical use. The HTS current feeder, which consists of current feedthroughs and/or current leads, can satisfy the requirement of stable large current transport capability with low heat load. The application to a magnetic-levitation coil is an example of other promising applications of HTS, which utilize the feature of high operating temperature.

\section{1.8 K HTS CURRENT FEEDTHROUGH}

\section{A. Development of 1.8 K HTS Current Feedthrough}

In the phase II experiment of the Large Helical Device (LHD) of the National Institute for Fusion Science (NIFS), it is planned to increase a plasma central field from $3 \mathrm{~T}$ to $4 \mathrm{~T}$ by cooling the helical coils from $4.4 \mathrm{~K}$ to $1.8 \mathrm{~K}$ using pressurized superfluid helium (He II) [1]. An HTS current feedthrough with high current capacity and low heat leak is one of the key components to be developed. It can connect $4.4 \mathrm{~K}$ and $1.8 \mathrm{~K}$ electrically. An YBCO bulk fabricated by quench and melt growth (QMG) process has been selected as a promising candidate material for the current feedthrough because of its large critical current density under the maximum stray magnetic field about $1 \mathrm{~T}$ in the LHD cryostat. 

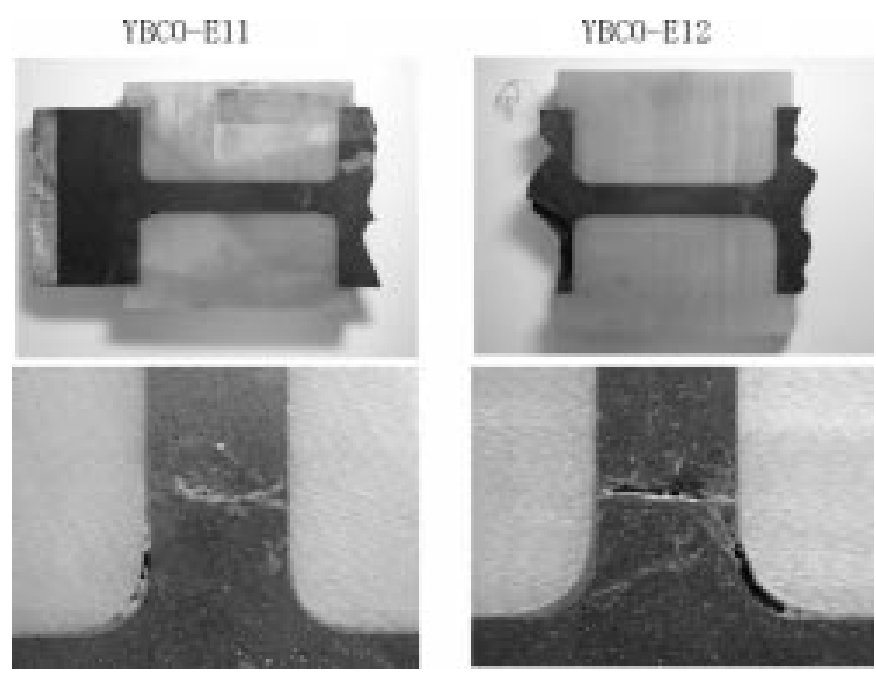

Fig. 2. Photographs of the broken YBCO conductors cut from the Prototype-I HTS current feedthrough.

TABLE I

THERMAL CONTRACTION FROM $300 \mathrm{~K}-4.2 \mathrm{~K}$ OF YBCO AND GFRP

\begin{tabular}{cccc}
\hline Direction & YBCO & $85 \%$ GFRP $(\perp)$ & $75 \%$ GFRP $(/ /)$ \\
\hline$X$ & $0.18 \%(\perp$ C-axis $)$ & $0.16 \%(/ /$ Glass $)$ & $0.19 \%(/ /$ Glass $)$ \\
$Y$ & $0.18 \%(\perp$ C-axis $)$ & $0.29 \%(\perp$ & $0.19 \%(/ /$ Glass $)$ \\
Glass $)$ & $0.16 \%(/ /$ Glass $)$ & $0.52 \%(\perp$ Glass $)$ \\
\hline & $\begin{array}{c}0.32 \%(/ / \\
\text { C-axis })\end{array}$ & & \\
\hline
\end{tabular}

Step-by-step developments have been carried out in NIFS as an collaboration with universities and laboratories mostly for Kyushu University. As a preliminary experiment to study the feasibility of YBCO bulk for the HTS current feedthrough, high current transport tests have been conducted with small " $\mathrm{H}$ " shaped samples (40 mm wide, $50 \mathrm{~mm}$ long, $7 \mathrm{~mm}$ thick, and cross-section of "-" part is $7 \mathrm{~mm} \times 7 \mathrm{~mm}$ ) [2]. The samples successfully carried current up to $25 \mathrm{kA}$ at $4.2 \mathrm{~K}$ and demonstrated a high current transport potential of the YBCO bulk. According to the feasibility study, we have tested four kinds of prototype HTS current feedthroughs described below.

\section{B. Prototype-I HTS Current Feedthrough}

Fig. 1 shows the first prototype HTS current feedthrough: prototype-I, which was designed for the large pressurized He-II test cryostat in the cryogenic laboratory of NIFS. A pair of the large " $\mathrm{H}$ " shaped YBCO bulk conductors $(60 \mathrm{~mm}$ wide, $140 \mathrm{~mm}$ long, $10 \mathrm{~mm}$ thick, and cross-section of the straight part "'-" is $10 \mathrm{~mm} \times 10 \mathrm{~mm}$ ), which were specially manufactured for this application, was embedded in a GFRP center plug of the $\lambda$-plate. Both sides of " $\mathrm{H}$ " shaped sample were soldered to copper electrodes. One pair of the electrodes was $4.4 \mathrm{~K}$ terminals (upper side) and the other pair was $1.8 \mathrm{~K}$ terminals (lower side). A pair of $1.8 \mathrm{~K}$ terminals was electrically shorted with $\mathrm{NbTi} / \mathrm{Cu} \mathrm{com}$ pacted strand cables for large current transport tests. During cool down from $300 \mathrm{~K}$ to $1.8 \mathrm{~K}$, however, one of the YBCO bulks broke mechanically due to the difference of thermal contraction between YBCO and GFRP. The other bulk also broke during warm up. Fig. 2 shows the photograph in which we cut the pro-

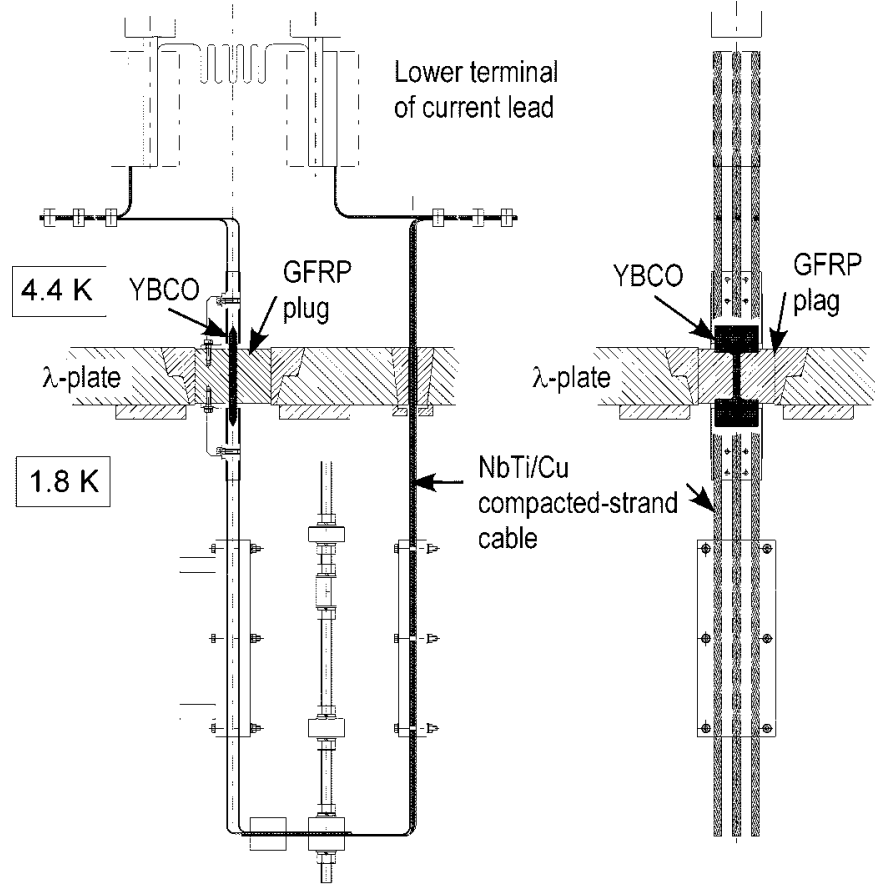

Fig. 3. Schematic drawing of the HTS current feedthrough: Prototype-II.

totype-I and observed the fracture part of the YBCO bulks. Both of the bulks were mechanically broken in the root section of " $\mathrm{H}$ " configuration buried into the GFRP plug. Therefore prototype-I could not be subjected to current transport tests. The thermal contractions of an YBCO bulk and GFRPs with different glass contents are listed in Table I. For the prototype-I, we used $85 \%$ glass content GFRP laminated in $Y$ direction whereas a $C$-axis of YBCO was oriented in $Z$ direction as shown in Fig. 1. After the cool down from $300 \mathrm{~K}$ to $4.4 \mathrm{~K}$, the YBCO bulk undertook the compressive force in $X$ and $Y$ directions, but it undertook the tensile force in $Z$ direction. Because the YBCO in the direction of $C$-axis easily comes off in thin sheets like mica, it was mechanically broken with the tensile force in $Z$ direction.

\section{Prototype-II}

To adjust the difference of the thermal contractions, the prototype-II was made with $75 \%$ glass content GFRP laminated in $Z$ direction. During cool down, the YBCO bulk undertook the compressive force in all directions. Fig. 3 shows the prototype-II HTS current feedthrough, in which only one YBCO bulk same size as that of the prototype-I was embedded in a GFRP center plug in order to reduce a risk and simplify the test. A current returning circuit was made by $\mathrm{NbTi} / \mathrm{Cu}$ compacted-strand cables penetrating $\lambda$-plate. The prototype-II was cooled successfully to $1.8 \mathrm{~K}$ without a mechanical damage in the bulk. The prototype-II could be passed by a current up to $500 \mathrm{~A}$. Then a pulse current of 5-10 kA suddenly flowed due to the misoperation of a power supply and it broke the bulk electrically. Fig. 4 shows that there was no damage of the bulk embedded in the GFRP but it was melting in the place just out of the GFRP. Since the time width of the pulse current was less than $1 \mathrm{~ms}$, it was suspected that the bulk melted, because a defect existed in the bulk from the beginning. 

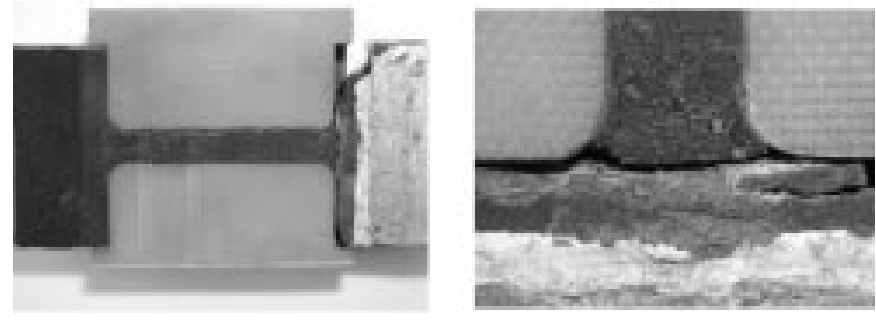

Fig. 4. Photographs of the broken YBCO conductors cut from the Prototype-II HTS current feedthrough.

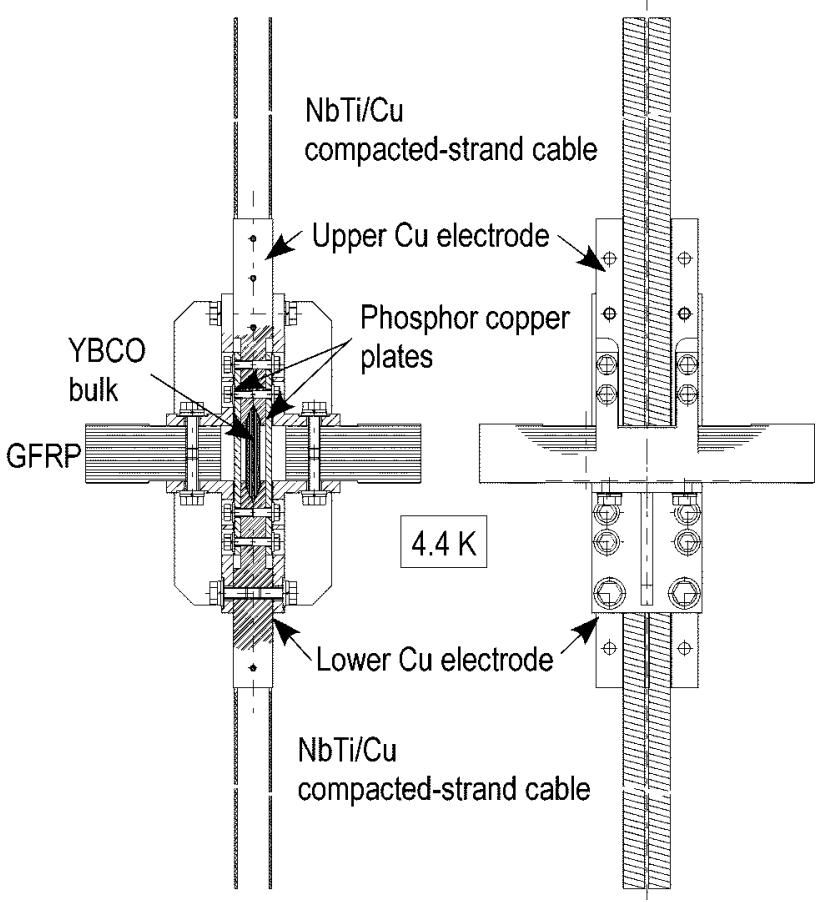

Fig. 5. Schematic drawing of the HTS current feedthrough: Prototype-IV.

\section{Prototype-III}

The prototype-III had the same design as the prototype-II and was tested in the normal liquid helium (He I) cryostat instead of the He II cryostat. Current transport experiments were conducted increasing the flat tops current gradually. A current of $1 \mathrm{kA}$ was reached successfully, at the current transport of $1.4 \mathrm{kA}$, the bulk broke electrically.

It was estimated that the cause of bulk fractures for the prototype-II and III was a defect inside the bulk from the beginning, which was not found by the inspection from the surface. It becomes clear that establishment of an inspection method for internal defect is indispensable to manufacture a large-sized YBCO bulk for the HTS current feedthrough.

\section{E. Prototype-IV}

The prototype-IV was manufactured from a bulk showing no defect inside by the magnetizing process before assembling. The component of Prototype-IV is shown in Fig. 5. The shape of the bulk was changed from the " $\mathrm{H}$ " type to the simple rectangular type to increase the mechanical strength. The increase of heat leak is negligible comparing to the heat generated at the

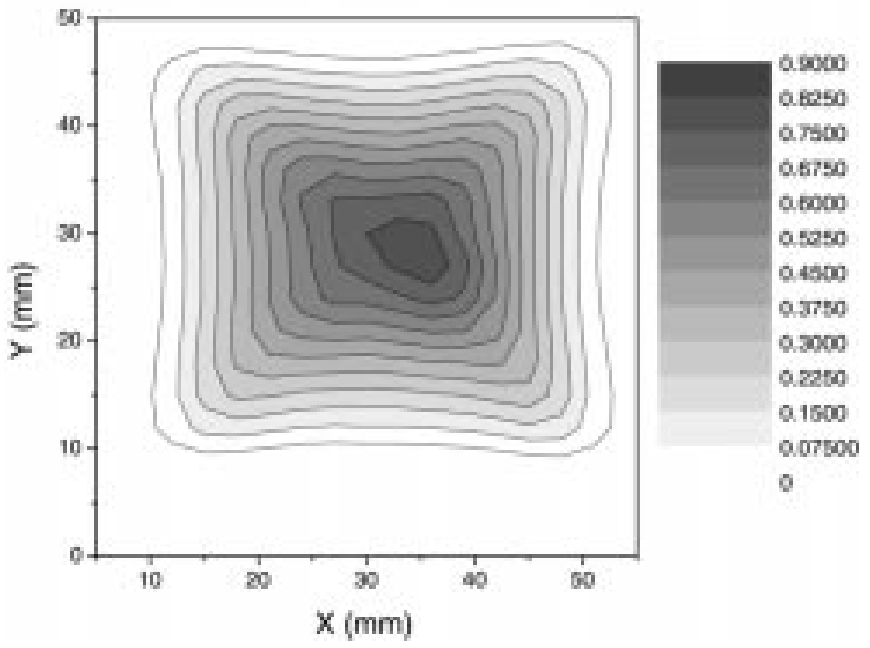

Fig. 6. Map of magnetization in the YBCO bulk to detect internal defects.

joint between an YBCO and a $\mathrm{Cu}$ electrode. The purpose of the prototype-IV was to check the inspection method and the manufacturing process. For it a small rectangular YBCO bulk (40 mm wide, $50 \mathrm{~mm}$ long and $7 \mathrm{~mm}$ thick) was used. In order to study the affection from the difference of thermal contraction between GFRP and YBCO step by step, in Prototype-IV, the bulk was not embedded into a GFRP. The phosphor copper plates of $3 \mathrm{~mm}$ thickness which serve both an electric by-pass and mechanical reinforcement have been arranged on both sides of the bulk, and they were fixed to the upper and lower copper electrodes. Cooling and current transport tests were conducted using the He I cryostat.

Fig. 6 shows the inspection result of the internal defect by the magnetizing process, which has been developed by the Nippon Steel Co. The magnetic field is first applied to the bulk by a magnet at the room temperature, and the magnetic field is lowered after cooling the bulk by liquid nitrogen. By measuring a surface magnetic field with a Hall probe, the map of the trapped flux density in the bulk can be obtained, which clarifies an internal defect. As shown in Fig. 6, magnetic flux was distributed in a smooth shape like a pyramid, and it has checked that internally there was no defect.

The prototype-IV was tested successfully in the trapezoidal wave current transport test up to $17.5 \mathrm{kA}$ as shown in Fig. 7. During the test of $20 \mathrm{kA}$, the NbTi/Cu compacted-strand cables, which were connecting the HTS current feedthrough and the conventional current lead, quenched at $19.6 \mathrm{kA}$, and it resulted in a thermal run away. In Fig. 7, no normal voltage was observed in the HTS bulk section, and there was no abnormality from the inspection after the warm up, either. The joint voltage did not have a difference between up-and-down connections, and it was confirmed that the solder joints were made under high quality control. The joint resistances are plotted as a function of the transport current in Fig. 8. They increased with the current and then reached constant values; $3.5 \mathrm{n} \Omega$ for the upper joint and $3.1 \mathrm{n} \Omega$ for the lower joint. The heat load from the joint resistance is about $1 \mathrm{~W}$ per joint at the transport current of $17.3 \mathrm{kA}$, which satisfies the requirement. 


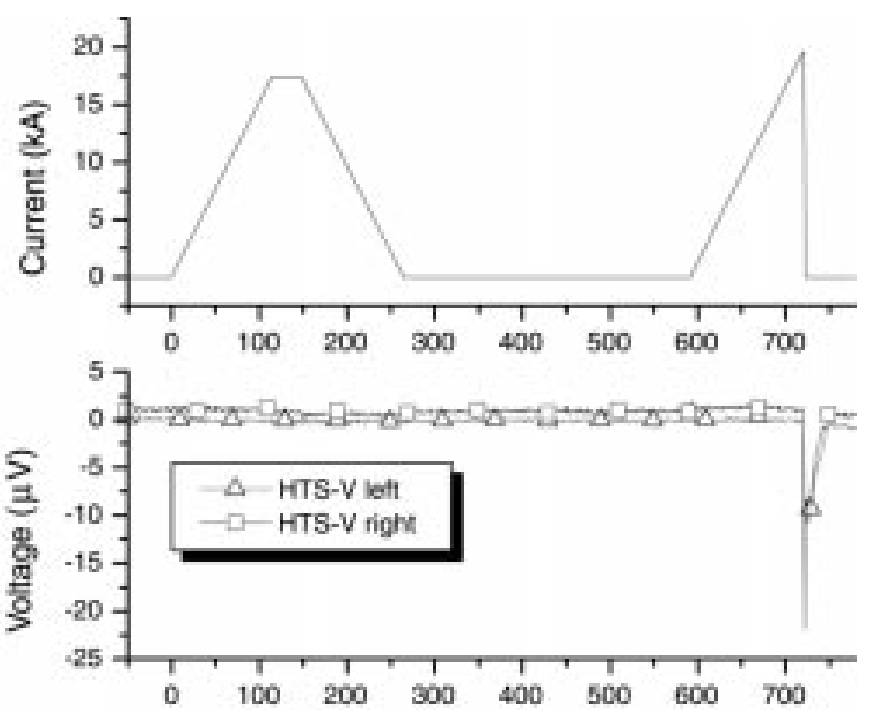

Fig. 7. Voltage drops in HTS part at the surfaces of left and right.

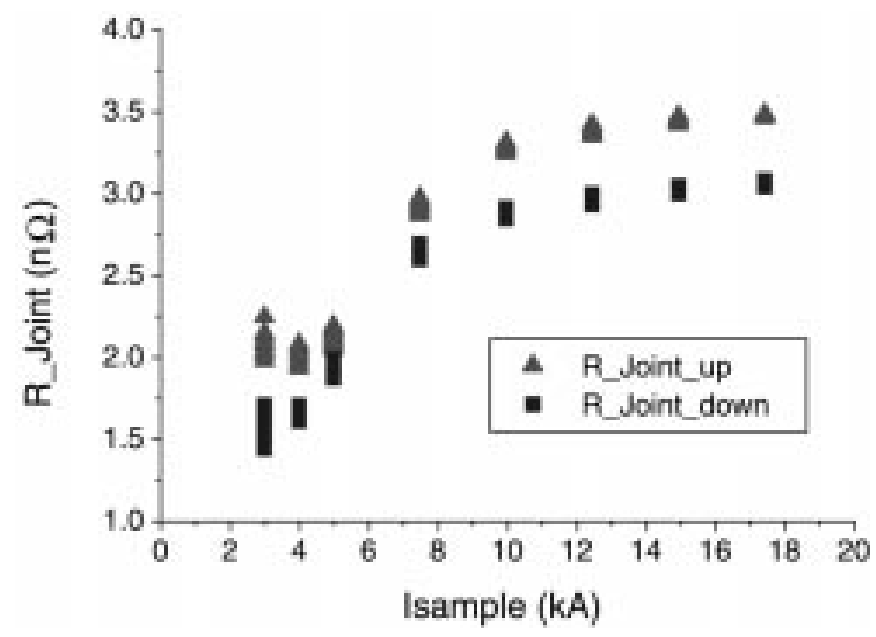

Fig. 8. Joint resistances of the prototype-IV HTS current feedthrough.

\section{F. Results of Prototype Experiments and a Future Program}

The effectiveness of the inspecting process to check an internal defect, before assembling the current feedthrough, has been shown by doing the magnetization measurement of the YBCO bulk at $\mathrm{LN}_{2}$ temperature. As a next step of experiment, the YBCO bulk which was used for prototype-IV is embedded into a GFRP, and same cooling and current-transport experiments are being conducted (prototype-V). Subsequently, a new prototype HTS current feedthrough, which uses two large-sized YBCO bulks of the thin strip shape with a width of $30 \mathrm{~mm}$, a length of $140 \mathrm{~mm}$, and a thickness of $10 \mathrm{~mm}$ to improve reliability, are planned to be manufactured and high current-transport tests in He I and He II will be carried out.

\section{HTS CURRENT LEAD}

The development of HTS current leads is progressing in the framework of the LHD International Mutual Experiment (LIME) with international universities and laboratories. A joint experiment of a $20 \mathrm{kA}$ HTS current lead developed by the Forschungszentrum Karlsruhe (FZK) was conducted in NIFS with FZK. The performance tests were successfully carried out and it demonstrated high potential of a HTS current lead using Ag/Au stabilized Bi-2223 tapes [3], [4].

$\mathrm{Bi}-2212$ bulk fabricated by the diffusion process is expected as an alternative material for HTS current leads. Development researches, such as large-current-transport experiment of this material and evaluation of mechanical property, are performed. The design study of a prototype HTS current lead, with mechanical reinforcement and current by-pass circuit, will be carried out [5], [6].

\section{HTS Magnetic-Levitation COIL}

The plasma experiment apparatus S-RT (Superconducting Ring Trap) is planned for the purpose of high beta plasma confinement research in the University of Tokyo. As a preceding step, Mini-RT, which is the size reduction version of S-RT, is under construction in collaboration with NIFS and Kyushu University. In this experiment a magnetic-levitation coil operated in persistent-current mode has to levitate for 8 hours without a feed of refrigerant. In the plasma vacuum vessel with $1000 \mathrm{~mm}$ bore, the magnetic-levitation coil with diameter of $300 \mathrm{~mm}$ is dominated by the levitation coil installed at the top of the vacuum vessel, and a position control is carried out. The coil winding has 435 turns of a Bi2223 tape wound on the brass bobbin furnished with the cooling pipe for indirect cooling. It is installed with the radiation shield into the coil vacuum vessel. Since a feed of refrigerant to the coil is impossible during the plasma experiment, the coil is designed so that the coil temperature rise after 8 hours levitation is less than $40 \mathrm{~K}$ with the specific heat of the coil and radiation shield. Therefore the heat loads to the coil and radiation shield are severely limited to $0.2 \mathrm{~W}$ and $0.78 \mathrm{~W}$, respectively. After the end of the daily plasma experiment, the coil is taken down to the maintenance location at the bottom of the plasma vacuum vessel, and the coil is re-cooled to $20 \mathrm{~K}$. In order to arrange the persistent current switch (PCS), the connectors for the detachable current leads and the check valves for the transfer lines, etc., we have to start with development of each component which is much smaller than the conventional one because the space in the coil vacuum vessel is strictly restricted. After the extensive design works, a full scale model for the HTS PCS has been successfully tested [7].

\section{SUMmary}

As a development program of the $20 \mathrm{kA}$ HTS current feedthrough from $4.4 \mathrm{~K}$ to $1.8 \mathrm{~K}$, four types of HTS current feedthroughs have been tested to evaluate their current transport capability in He II and He I cooling condition. During cool down from $300 \mathrm{~K}$ to $1.8 \mathrm{~K}$, the YBCO bulks of prototype-I broke mechanically due to the difference of thermal contraction between YBCO and GFRP. The prototype-II and III broke electrically due to a defect inside the bulk, which was not found by the inspection from the surface. In tests of the prototype-IV, effectiveness of the inspecting process to check an internal defect, before assembling, has been shown by doing the magnetization measurement of the $\mathrm{YBCO}$ bulk at $\mathrm{LN}_{2}$ temperature. 
As a development of the HTS current lead, large-currenttransport experiment of a large-size Bi2212 bulk fabricated by the diffusion process are being performed.

As a development of the HTS magnetic-levitation coil for the Mini-RT, a full scale model of the HTS PCS has been successfully tested. By the end of 2001, a simple test of the magnetic-levitation coil will be carried out.

\section{ACKNOWLEDGMENT}

The authors wish to express their thanks to Fuji Electric Co., Ltd., for their collaboration to develop the HTS current feedthrough and the HTS magnetic-levitation coil. They would like to thank Nippon Steel Co. for their works to develop a large-sized YBCO bulk for the HTS current feedthrough.

\section{REFERENCES}

[1] T. Mito et al., "Developments of high-Tc superconducting current feeders for a large-scale superconducting coil system," J. Nucl. Mater. 258-263, pp. 1940-1945, 1998.

[2] K. Maehata et al., "High current transport test of a YBCO bulk conductor up to 25 kA," IEEE Trans. Appl. Superconduct., vol. 9, no. 2, pp. 1281-1284, 1999.

[3] R. Heller et al., "Test results of a $20 \mathrm{kA}$ current lead using Ag/Au stabilized Bi-2223 tapes," IEEE Trans. Appl. Superconduct., vol. 11, no. 1, pp. 2603-2606, 2001.

[4] T. Mito et al., "Development of high temperature superconducting current feeders for a large-scale superconducting experimental fusion system," IEEE Trans. Appl. Superconduct., vol. 11, no. 1, pp. 2611-2614, 2001.

[5] Y. Yamada et al., "Performance test of Bi-2212 HTS current leads prepared by the diffusion process," in MT-17, 2001, TUPO3D2-09.

[6] H. Tamura et al., "Mechanical properties and reinforcement of Bi2212 cylindrical bulk superconductor for current lead," in $M T-17,2001$, TUPO3D2-08.

[7] N. Yanagi et al., "Engineering research and development for magnetically-levitated high-temperature superconducting coil system of Mini-RT project," in MT-17, 2001, MOPO2A4-13. 